\title{
On the Suitability of Employing Silicon Photomultipliers for Underwater Wireless Optical Communication Links
}

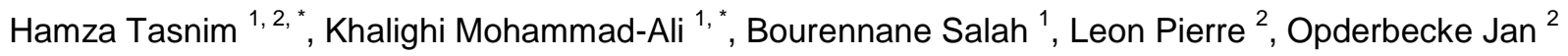 \\ ${ }^{1}$ Aix Marseille Univ, CNRS, Inst Fresnel, Cent Marseille, Marseille, France. \\ ${ }^{2}$ IFREMER Res Inst, La Seyne Sur Mer, France. \\ * Corresponding authors : Tasnim Hamza, email address : tasnim.hamza@fresnel.fr ; Mohammad-Ali \\ Khalighi, email address : ali.khalighi@fresnel.fr
}

\begin{abstract}
:
We investigate the use of Silicon photomultipliers (SiPMs) for signal detection in underwater wireless optical communication (UWOC) systems. We present an analytical photon counting model for the SiPM and clarify the related noise sources. Then, for a typical UWOC link, we compare the link performance for the two cases of using an SiPM and an avalanche photo-diode, and show to which extent the link span can be increased for the case of an SiPM-based receiver. The results we present testify the practical interest and the feasibility of employing SiPMs in the UWOC context.
\end{abstract}

Keywords : Underwater wireless optical communications, photo detection, Silicon photomultiplier, multipixel photon counter, single-photon avalanche diode 


\section{INTRODUCTION}

Underwater wireless optical communication (UWOC) is considered today as a reliable and promising technology for establishing high data rate links in sub-sea environments. Despite this high potential, its deployment is currently limited to relatively short-to-moderate link spans, typically of several tens of meters in clear waters [1], [2].

Whereas light emitting diodes (LEDs) are mostly used at the transmitter (Tx) in UWOC systems for the reasons of low cost and beam alignment simplicity, the choice of photo-detector (PD) at the receiver (Rx) remains debatable. The classical PIN PDs appear to be an unsuitable choice due to high attenuation in the aquatic medium and are challenged by avalanche photo-diodes (APDs), interesting due to their inherent gain. APDs have been considered in several previous experimental and theoretical works, e.g. [3], [4]. Photo-multiplier tubes (PMTs) appear to be a more suitable choice in this context and have drawn particular attention thanks to their high sensitivity to very low intensity signals (down to a few photons) and high gain [2], [3]. Nevertheless, they are bulky and more expensive, require high voltage for operation, and are more easily damaged by exposure to high-intensity light [5].

Very recently, silicon photo-multipliers (SiPMs), also known as multi-pixel photon counters (MPPCs), have emerged as an interesting photo-detection solution when working with very low intensity levels down to a single photon [6]. SiPMs have been reported to offer several advantages such as low operation voltage, insensitivity to magnetic fields and mechanical robustness (unlike PMTs). The reflection about using SiPMs in optical communications was initiated in [7], [8], where the feasibility of their use as PD was proven. SiPMs were later employed in several works in the domain of optical communications. In [9] these devices were considered for the design of inexpensive yet very sensitive receivers for plastic optical fiber links, capable of detecting small number of photons over long distances. In [10], [11], [12], SiPMs were investigated in the context of long-distance and low-power visible light communications (VLC) together with optical orthogonal-frequency-division-multiplexing (OFDM) signaling; for instance, for down-hole monitoring applications. In particular, an experimental work in [13] demonstrated a $200 \mathrm{Mbps}$ data rate VLC link using a SPAD-based Rx.

In this work, we investigate the interest and feasibility of employing SiPMs in UWOC applications. Considering a typical link configuration, we evaluate the performance of an SiPM-based Rx and illustrate its advantage, compared to the case of using an APD. Given these results, we discuss the relevance and reliability of using SiPMs in the UWOC context. In fact, as we will explain latter in Section III, an SiPM is composed of a number of APDs biased above the breakdown voltage, whereas in the classical APD use, the PD is biased below its breakdown voltage. Due to this reason, we consider the APD case as benchmark in our study of SiPMs.

The remainder of the paper is organized as follows. In Section II, we present the UWOC system description including channel model and the Rx noise for the APD case. Next, details on SiPM Rx modelling are provided in Section III and some numerical results are provided in Section IV to evaluate the Rx performance. Section V concludes the paper.

\section{MAIn ASSUMPTIONS AND SYSTEM MODEL}

We consider a point-to-point communication link, as depicted in Fig. 1 with a LED-based Tx. Without loss of generality, we model the LED radiation pattern $P_{\mathrm{t}}$ by a generalized Lambertian model with azimuthal symmetry, as follows:

$$
P_{\mathrm{t}}=P_{\mathrm{Tx}} \frac{m+1}{2 \pi} \cos ^{m}(\theta) \quad[\mathrm{W} / \mathrm{sr}]
$$




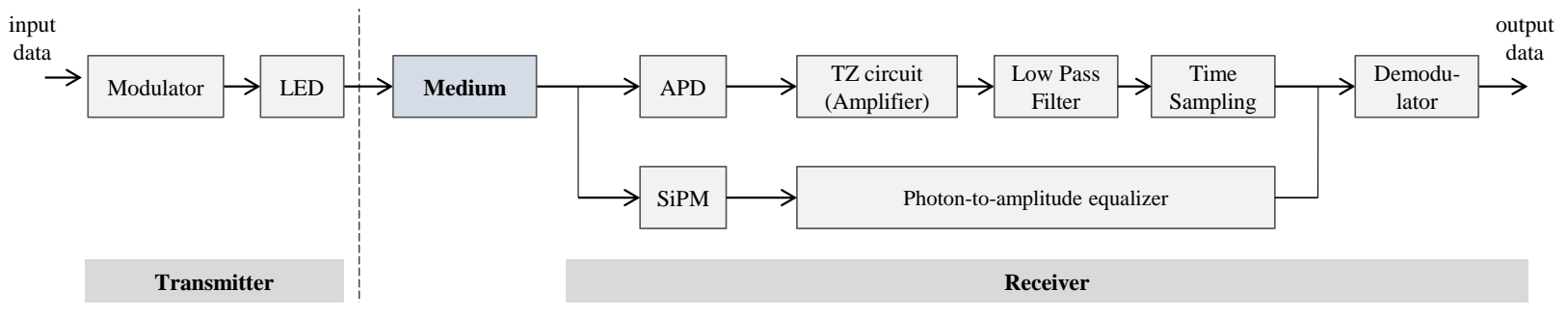

Fig. 1. End-to-End general block diagram of the UWOC communication link.

where $P_{\mathrm{Tx}}$ denotes the transmit power, $\theta \in[0, \pi / 2]$ is the angle of irradiance, and $m$ is the Lambertian order given by $m=-\ln (2) / \ln \left(\cos \left(\theta_{1 / 2}\right)\right)$, with $\theta_{1 / 2}$ being the Tx semi-angle at half-power. The LED is considered to emit at $\lambda_{0}=470 \mathrm{~nm}$ with a Lambertian order of 45 , corresponding to $\theta_{1 / 2} \approx 10^{\circ}$.

We conduct our study for the case of clear ocean waters with an attenuation coefficient of $c=0.15 \mathrm{~m}^{-1}$ [14]. We model the optical power loss by the Beer-Lambert law, which has been demonstrated as a good approximation of the channel power loss for the case of low turbidity waters [14]. This way, assuming perfect Tx-Rx alignment, the captured power by the $\mathrm{Rx}, P_{\mathrm{Rx}}$, is given by [15]:

$$
P_{\mathrm{Rx}}=P_{\mathrm{t}} \exp (-c Z) \frac{A_{\mathrm{PD}}}{Z^{2}},
$$

where $Z$ is the distance between the Tx and the $\mathrm{Rx}$ and $A_{\mathrm{PD}}$ is the physical area of the PD.

Concerning the Rx noise for the case of an APD, we take into account the photo-current shot noise, arising from the input signal. We reasonably neglect the shot noise arising from background radiations, assuming that our system operates in deep waters. We also take into account the thermal noise, mostly generated by the load resistor of the trans-impedance (TZ) circuitry, refer to Fig.1. Note that the TZ amplifier is useless for the SiPM case and is used only for the APD case, see the next section. We reasonably neglect the dark current noise assuming a Silicon PD [16], [17]. The reader is referred to [16] for a complete model of the $\mathrm{Rx}$ noise in this case.

\section{SIPM-BASED RECEIVER}

An SiPM is an array of APD pixels (also called microcells) operating in Geiger mode; also called single photon avalanche diodes (SPADs). In this operation mode, diodes are biased above the breakdown voltage. When a photon heats the SPAD sensitive area, an avalanche breakdown is triggered and leads to the generation of an output pulse. Thus, the SPAD is considered as a single photon counter [11] and the SiPM output is the sum of single SPADs' photon counting. Each SPAD is connected to a quenching device that halts the Geiger discharge. Depending on its type, the SPAD (and therefore the $\mathrm{SiPM}$ ) is said to be actively or passively quenched.

\section{A. Performance parameters}

The performance of an SiPM depends on several parameters, including its photon detection efficiency
(PDE) that we denote by $\Upsilon_{\mathrm{PDE}}$. It is defined as the product of the quantum efficiency, the fill factor (ratio of the active area to the total physical area of the PD), and the probability that a detected photon will generate an avalanche. The SiPM is affected by several sources of noise. The first one consists of after-pulsing (AP), defined as the re-trigger of the SPAD by trapped carriers at the SPAD output from the previous avalanche. We denote the probability of AP by $P_{\mathrm{AP}}$. Another noise source is the cross-talk (CT), which occurs when an avalanching micro-cell initiates an avalanche in another micro-cell and is defined by the probability $P_{\mathrm{CT}}$. The main source of SiPM noise is the dark count rate (DCR) which results from the breakdown of the micro-cell due to thermally generated electrons, that we denote by $f_{\mathrm{DCR}}$. Another key parameter of an SiPM is the micro-cell dead-time $\left(\tau_{\mathrm{d}}\right)$, also known as the recovery time. It is the time needed by a micro-cell to recharge and during which it is unable to detect an arriving photon.

\section{B. SiPM photon counting}

We denote by $C_{\mathrm{ph}}$ the output photon count of the SiPM, which is equal to the sum of individual counts from the SPADs, $c_{\mathrm{ph}}(i)$, as follows:

$$
C_{\mathrm{ph}}=\sum_{i=1}^{N_{\mathrm{SPAD}}} c_{\mathrm{ph}}(i),
$$

where $N_{\text {SPAD }}$ denotes the number of SPAD pixels forming the SiPM. We assume that the SiPM has an ideal photon counter behaviour and model its photo-detection by a Poisson distribution [12] as follows:

$$
\operatorname{Pr}\left(C_{\mathrm{ph}}=k\right)=\exp (-\mu) \frac{\mu^{k}}{k !}
$$

Here, $\mu$ denotes the average photon count and is expressed as a function of the captured optical power by the Rx as follows [10]:

$$
\mu=\left(\frac{\Upsilon_{\mathrm{PDE}} P_{\mathrm{Rx}}}{E_{\mathrm{ph}}}+f_{\mathrm{DCR}}\right)\left(1+P_{\mathrm{AP}}+P_{\mathrm{CT}}\right) T,
$$

where $T$ denotes the bit time and $E_{\mathrm{ph}}=\hbar c / \lambda$ is the energy of photon, with $\hbar, c$, and $\lambda$ being the Planck constant, the speed of light, and the light wavelength, respectively.

It has been shown in [12], that the SiPM generates a non-linear distortion (NLD) on the signal resulting from the saturation of the SPADs. This saturation is 
observed when photon arrivals occur during the SPAD dead-time. The related SPAD behavior depends further on the quenching device. Indeed, for the case of passive quenching, the extra-photon arrivals, although uncounted, extend the pixel dead-time, causing the SPAD to collapse. For this reason, the passively quenched SPAD are said to be paralyzable detectors [12]. On the other hand, for the case of active quenching, the incoming photons during the dead-time are not counted and do not prolong the deadtime. Hence, the actively quenched SPADs are said to be non-paralyzable detectors [12]. Given (5), the average output of the SiPM during a bit time $T$ is expressed as a function of $\mu$ as follows [12]:

$$
\begin{aligned}
& \mu_{\mathrm{PQ}}=\mu \exp \left(-\frac{\mu \tau_{\mathrm{d}}}{T N_{\mathrm{SPAD}}}\right) \\
& \mu_{\mathrm{AQ}}=\frac{\mu}{1+\frac{\mu \tau_{\mathrm{d}}}{T}},
\end{aligned}
$$

where $\mu_{\mathrm{PQ}}$ and $\mu_{\mathrm{AQ}}$ denote the average photon counts for the cases of passively quenched (PQ) and actively quenched (AQ) SiPM, respectively.

Considering the SPAD's dead-time constraint, an SiPM is practically able to detect photons at a maximum rate of $R_{\max }=1 / \tau_{d}$ [18]. For this reason, in our study we fix the data transmission rate $R$ such that $R \leq R_{\max }$.

\section{BER analysis}

In this study, we consider intensity modulation with direct detection (IM/DD), based on non-return to zero (NRZ) on-off keying (OOK) modulation as the transmission scheme, which has the advantage of implementation simplicity. Let us denote by $P_{0}$ and $P_{1}$ the transmitted optical power for bits ' 0 ' and ' 1 ', respectively. Referring to (2) and (5)-(7), we calculate the corresponding average number of received photons, that we denote by $\mu_{0}$ and $\mu_{1}$, respectively. For OOK demodulation at the Rx, we define the detection threshold $\mu_{\mathrm{th}}$ as follows:

$$
\mu_{\mathrm{th}}=\frac{\mu_{0}+\mu_{1}}{2} \text {. }
$$

Thus, the error probability $P e$, can be calculated as follows [10]:

$$
\begin{aligned}
P e & =\frac{1}{2} \operatorname{Pr}\left(C_{\mathrm{ph}} \leq \mu_{\mathrm{th}} \mid \mu=\mu_{1}\right)+\frac{1}{2} \operatorname{Pr}\left(C_{\mathrm{ph}}>\mu_{\mathrm{th}} \mid \mu=\mu_{0}\right) \\
& =\frac{1}{2}\left[1-F_{\mathrm{c}}\left(\mu_{\mathrm{th}}, \mu_{0}\right)+F_{\mathrm{c}}\left(\mu_{\mathrm{th}}, \mu_{1}\right)\right],
\end{aligned}
$$

where $F_{\mathrm{c}}(x, y)$ denotes the cumulative distribution function of the Poisson-distributed random variable $x$ with parameter $y$.

\section{NumeriCAL RESUlTS}

In this section, we present some simulation results in order to study the performance of an SiPM-based $\mathrm{Rx}$ for UWOC links. The Rx simulation parameters are specified in Table I. These parameters correspond to a SensL B-series 30020 SiPM PD based Rx [19]. Here, we take $R=3 \mathrm{Mbps}$, which can be readily attained using the typical off-the-shelf available LEDs. We assume that the considered SiPM and APD have equal physical areas. Note that, whereas the physical area of an APD is

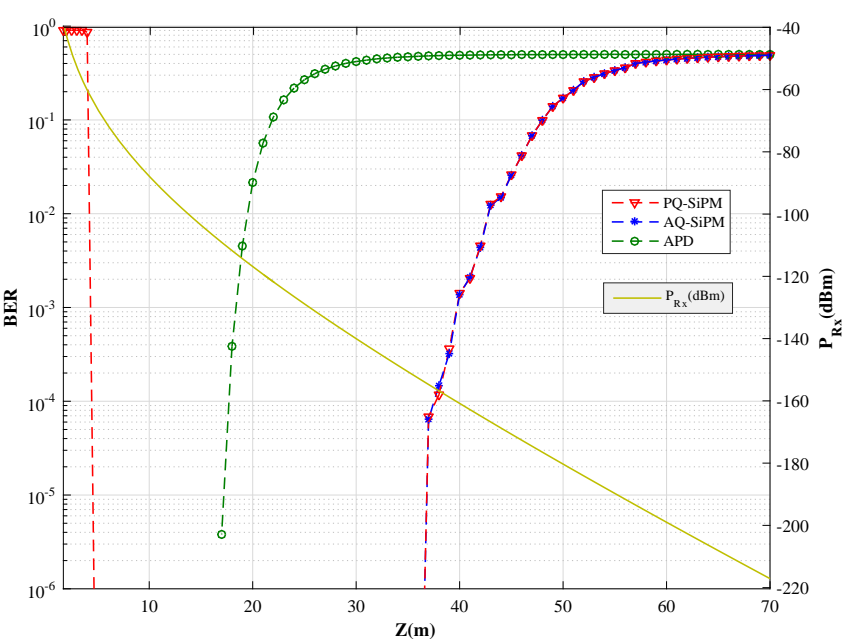

Fig. 2. BER performance and $P_{\mathrm{Rx}}(\mathrm{dBm})$ as a function of link range $Z$ for AQ- and PQ-SiPM, and APD-based Rx. $P_{1}=1 \mathrm{~W}$, EXT $=0$, uncoded NRZ-OOK modulation, data rate $R=3 \mathrm{Mbps}$. System operating in clear ocean waters with $c=0.15 \mathrm{~m}^{-1}$.

almost totally photo-sensitive, the fill factor of an SiPM is typically on the order of $40 \%$.

TABLE I

RX PARAMETER SPECIFICATION

\begin{tabular}{|l|l|}
\hline Parameter & Value \\
\hline \hline SiPM PDE, $\Upsilon_{\mathrm{PDE}}$ & 0.31 \\
SiPM area & $9 \mathrm{~mm}^{2}$ \\
SiPM Dark Count Rate, $f_{\mathrm{DCR}}$ & $6.6 \mathrm{MHz}$ \\
SPAD dead-time, $\tau_{\mathrm{d}}$ & $90 \mathrm{~ns}$ \\
Number of SPAD in SiPM, $N_{\mathrm{SPAD}}$ & 10998 \\
Probability of Cross-Talk, $P_{\mathrm{CT}}$ & 0.1 \\
Probability of After-Pulsing, $P_{\mathrm{AP}}$ & 0.01 \\
\hline \hline APD load resistance, $R_{\mathrm{L}}$ & $50 \Omega$ \\
APD gain, $G$ & 50 \\
APD ionization ratio, $\xi$ & 0.02 \\
\hline
\end{tabular}

We first set the extinction ratio $\mathrm{EXT}=P_{0} / P_{1}$ to 0 . We have presented the link BER performance of the cases of PQ- and AQ-SiPM and APD as a function of the link span $Z$ in Fig. 2. We have also shown the plot of the received power, $P_{\mathrm{Rx}}$.

Let us consider a target BER of $10^{-4}$. From the results in Fig. 2, an SiPM-based Rx achieves a communication link span around $36 \mathrm{~m}$, for both cases of AQ and PQ detectors. On the other hand, the APD-based link distance is limited to about $17 \mathrm{~m}$ only. The corresponding received power $P_{\mathrm{Rx}}$ for $Z=17 \mathrm{~m}$ and $36 \mathrm{~m}$ are on the order of $-110 \mathrm{dBm}$ and $-152 \mathrm{dBm}$, respectively, which shows the capability of SiPMs to work under very low signal levels compared to APDs. Note that the suitability of SiPM to UWOC systems comes especially along with the use of LEDs as the Tx emitting source. Indeed, LEDs have typically a much larger beam divergence compared to laser diodes, which results in a higher geometric loss, and hence much reduced link span; the price paid for simpli- 


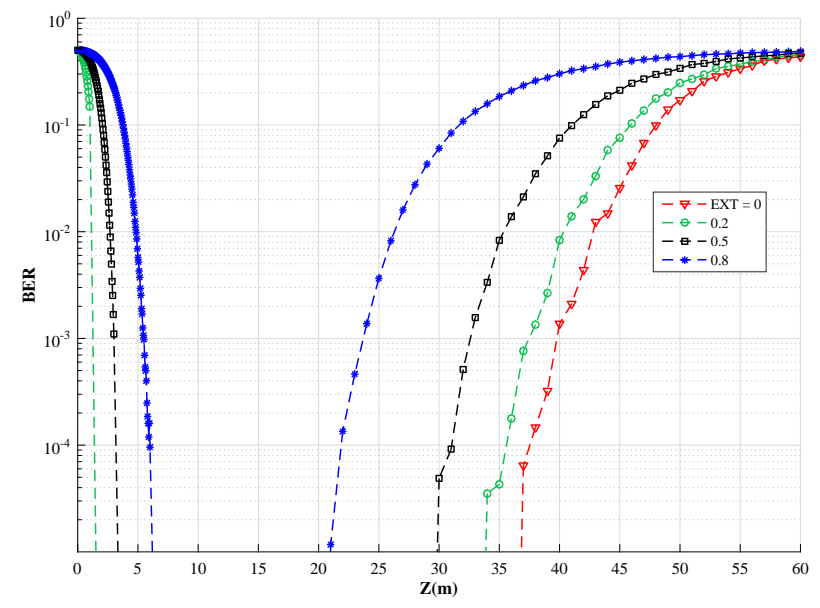

(a) AQ-SiPM

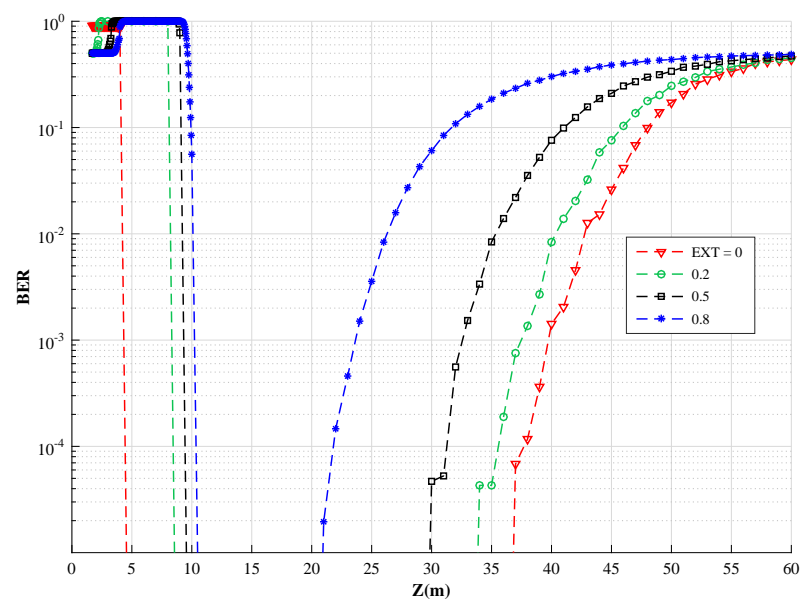

(b) PQ-SiPM

Fig. 3. BER performance as a function of link range $Z$ for AQ- and PQ-SiPM-based Rx, considering different EXT. $P_{\mathrm{t}}=1 \mathrm{~W}$, uncoded NRZ-OOK modulation, $R=3 \mathrm{Mbps}$, clear ocean waters with $c=0.15 \mathrm{~m}^{-1}$.

fied Tx-Rx alignment. The use of an SiPM-based Rx is then particularly promising and enables data transmission over considerably increased link spans, compared to an APD-based Rx.

Fig. 2 further shows that NLD arises for the case of PQSiPM for $P_{\mathrm{Rx}} \geq 66 \mathrm{dBm}$, corresponding to $Z \leq 5 \mathrm{~m}$, which is the result of detector saturation. So, unless for too short link distances (rather unlikely to happen in practice), the PQ-SiPM is not concerned by NLD problem. We do not notice this behavior for the case of an AQ-SiPM. However, this is valid for the special case of $\mathrm{EXT}=0$ that we consider for Fig. 2 results.

In order to get more insight into the impact of NLD on the link performance, let us investigate the case were EXT $\neq 0$, which is mostly the case in practice because the minimum intensity of a LED is never equal to zero. The simulation results are presented in Fig.3(a) and Fig. 3(b) for the cases of AQ- and PQ-SiPM, respectively. We notice that the SiPM performance strongly depends on the EXT parameter. Indeed, when we rise the EXT from 0 to 0.8 , the maximum achievable link span for a target BER of $10^{-4}$ is reduced from $\sim 36 \mathrm{~m}$ to $\sim 21 \mathrm{~m}$ for both AQ- and PQ-SiPM cases. This could be expected since increasing the EXT means that the power levels corresponding to bits ' 0 ' and ' 1 ' are closer to each other, resulting in a higher error probability according to (9). We also notice that the NLD effect is manifest at a larger $Z$ as EXT increases, and this is also valid for the case of AQ-SiPM, although we noticed an NLD-free performance for $\mathrm{EXT}=0$. For instance, for EXT $=0.8$, the NLD limitation occurs at $Z \leq 6 \mathrm{~m}$ for the case of AQ-SiPM, while this lower limit of the link span increases to about $11 \mathrm{~m}$ for the case of PQ-SiPM. We conclude that, overall, not only a relatively high EXT ratio reduces significantly the link span for a target BER, it also impacts the range of $Z$ over which SiPM operates in the NLD-free (i.e., linear) mode.

\section{CONCLUSIONS}

In this paper, we demonstrated the feasibility of using SiPM detectors in UWOC systems. We showed that these PDs provide significant performance improvement compared to the case of APDs, when working with very low signal intensity levels, e.g. for long link spans. In particular, we studied the effect of non-linear distortion generated by the SiPM operation mode and showed that its impact on the link performance and range strongly depends on the LED extinction ratio. In fact, increasing the EXT parameter reduces the operation flexibility in terms of the maximum and minimum achievable link spans for a given target BER performance. Meanwhile, we showed that the limitation due to non-linear distortion occurs in very short link ranges,e.g. lower than a few meters for EXT $<0.5$.

\section{ACKNOWLEDGMENT}

This work was supported in part by the French PACA (Provence, Alpes, Côte d'Azur) Regional Council. The authors wish to thank Prof. Hassan Akhouayri and Dr. Frédéric Lemarquis from Institut Fresnel and Dr. Majid Safari from University of Edinburgh for the fruitful discussions on the practical considerations concerning the transmitter/receiver opto-electronics.

\section{REFERENCES}

[1] M. A. Khalighi, C. Gabriel, T. Hamza, S. Bourennane, P. Léon, and V. Rigaud, "Underwater wireless optical communication; recent advances and remaining challenges," pp. 1-4, July 2014, Graz, Austria.

[2] F. Hanson and S. Radic, "High bandwidth underwater optical communication," Applied Optics, vol. 47, no. 2, pp. 277-283, Jan. 2008.

[3] B. Cochenour, L. Mullen, and J. Muth, "Temporal response of the underwater optical channel for high-bandwidth wireless laser communications," IEEE Journal of Oceanic Engineering, vol. 38 , no. 4, pp. 730-742, Oct. 2013.

[4] M. Doniec, M. Angermann, and D. Rus, "An end-to-end signal strength model for underwater optical communications," IEEE Journal of Oceanic Engineering, vol. 38, no. 4, pp. 743-757, Oct. 2013. 
[5] P. Lacovara, "High-bandwidth underwater communications," $M a$ rine Technology Society Journal, vol. 42, no. 1, pp. 93-102, Mar 2008 .

[6] SensL, "Intoduction to the Silicon Photomultiplier, Technical Note," http://www.sensl.com/, 2011.

[7] I. Alsolami, D. Chitnis, D. C. O’Brien, and S. Collins, "Broadcasting over photon-counting channels via multiresolution ppm Implementation and experimental results," IEEE Communications Letters, vol. 16, no. 12, pp. 2072-2074, Dec 2012.

[8] G. Zhang, C. Yu, C. Zhu, and L. Liu, "Feasibility study of MultiPixel Photon Counter serving as the detector in digital optical communications," Optik - International Journal for Light and Electron Optics, vol. 124, no. 22, pp. 5781 - 5786, 2013.

[9] D. Chitnis and S. Collins, "A SPAD-Based Photon Detecting System for Optical Communications," Journal of Lightwave Technology, vol. 32, no. 10, pp. 2028-2034, May 2014

[10] Y. Li, S. Videv, M. Abdallah, K. Qaraqe, M. Uysal, and H. Haas, "Single photon avalanche diode (SPAD) VLC system and application to downhole monitoring," in Global Communications Conference (GLOBECOM), 2014 IEEE, Dec 2014, pp. 2108-2113.

[11] Y. Li, M. Safari, R. Henderson, and H. Haas, "Optical OFDM with single-photon avalanche diode," Photonics Technology Letters, IEEE, vol. 27, no. 9, pp. 943-946, May 2015.

[12] Y. Li, M. Safari, R. Henderson, and H. Haas, "Nonlinear distortion in SPAD-based optical OFDM systems," Global Telecommunications (Globecom) Conference, Workshop on Optical Wireless Communications, Dec. 2015, San Diego, CA, USA.
[13] D. Chitnis, L. Zhang, H. Chun, S. Rajbhandari, G. Faulkner, D. O'Brien, and S. Collins, "A $200 \mathrm{Mb} / \mathrm{s}$ VLC demonstration with a SPAD based receiver," in 2015 IEEE Summer Topicals Meeting Series (SUM), July 2015, pp. 226-227.

[14] C. Gabriel, M. A. Khalighi, S. Bourennane, P. Léon, and V. Rigaud, "Monte-carlo-based channel characterization for underwater optical communication systems," IEEE/OSA Journal of Optical Communications and Networking, vol. 5, no. 1, pp. 1-12, Jan. 2013.

[15] M. Kavehrad, M. I. S. Chowdhury, and Z. Zhou, Fundamentals of Optical Wireless Communications, in Short-Range Optical Wireless: Theory and Applications. Chichester, UK: John Wiley and Sons, Ltd, 2015.

[16] F. Xu, M. A. Khalighi, and S. Bourennane, "Impact of different noise sources on the performance of PIN- and APD-based FSO receivers," COST ICO802 Workshop, IEEE ConTEL Conference, pp. 211-218, June 2011, Graz, Austria

[17] R. Toledo-Crow, S. Shi, and Y. Li, "Analysis of a two-PMT system for simultaneous back- and forward-fluorescence detection in multiphoton microscopy," Biomedical Optics, Optical Society of America, p. BMD56, 2008.

[18] S. Donati and T. Tambosso, "Single-photon detectors: From traditional pmt to solid-state spad-based technology," IEEE Journal of Selected Topics in Quantum Electronics, vol. 20, no. 6, pp. 204211, Nov 2014.

[19] SensL, "B-Series Fast, Blue-Sensitive Silicon Photomultiplier Sensors Datasheet," http://www.sensl.com/, 2013. 